\title{
On the role of pre-existing, unhealed cracks on the bending strain response of $\mathrm{Ag}$-clad $(\mathrm{Bi}, \mathrm{Pb})_{2} \mathrm{Sr}_{2} \mathrm{Ca}_{2} \mathrm{Cu}_{3} \mathrm{O}_{x}$ tapes
}

\author{
M. Polak, ${ }^{\text {a) }}$ J. A. Parrell, A. A. Polyanskii, ${ }^{\text {b) }}$ A. E. Pashitski, and D. C. Larbalestier \\ Applied Superconductivity Center, University of Wisconsin, Madison, Wisconsin 53706
}

(Received 26 August 1996; accepted for publication 12 December 1996)

\begin{abstract}
Studies of the transport critical current $\left(I_{c}\right)$, magnetization, magnetic flux penetration, and microstructure of pressed and rolled Ag-clad $(\mathrm{Bi}, \mathrm{Pb})_{2} \mathrm{Sr}_{2} \mathrm{Ca}_{2} \mathrm{Cu}_{3} \mathrm{O}_{x}$ tapes (2223) have been made as a function of bending strain. Pressed tapes exhibited markedly less degradation of $I_{c}$ from strain than did rolled tapes, while the magnetization of pressed tapes declined much more rapidly with bending strain than did either of the transport currents. Magneto-optical imaging of nonbent pressed samples revealed a network of flux-penetrated defect channels that were primarily oriented parallel to the tape axis. Bending such samples to a small strain increased the visibility of these defects, believed to be cracks. This network correlates well to the cracks produced in intermediate thermomechanical processing deformation steps. The greater sensitivity of the transport current of rolled samples to bending is further direct proof of the fact that the tape "remembers" the cracks induced in the core during intermediate deformation and that heat treatment after the deformation does not heal all damage. (C) 1997 American Institute of Physics. [S0003-6951(97)00608-6]
\end{abstract}

Many factors can limit the critical current density $\left(J_{c}\right)$ of polycrystalline high temperature superconductors. ${ }^{1,2}$ Among the most difficult to quantify are microcracks, pores, and other defects limiting the connectivity of the polycrystalline superconductor. Such defects are believed to be a major factor limiting the critical current density $\left(J_{c}\right)$ of Ag-clad BSCCO-2223 tapes ${ }^{3-8}$ but specific proof of this point is lacking because establishing the residual crack density of deformed-and-reacted BSCCO tapes is extremely hard. Recently Parrell et al. ${ }^{8}$ used magneto-optical (MO) imaging to show the significant and differing effects of residual cracks (i.e., those left even after subsequent heat treatment) in rolled and pressed tapes. Clear evidence was seen that flux tended to enter the tape in narrow channels which were preferentially oriented along the long axis for pressed tapes and transverse to the axis for rolled tapes. These directions are coincident with the principal direction of thermomechanical processing deformation cracking. ${ }^{3,6}$ However, magnetooptical images are sensitive to all defects which admit flux, including weak-linked grain boundaries that are otherwise physically connected. To develop more specific knowledge about cracks alone, we have conducted an experiment in which we deliberately cracked a series of tapes by bending. We have examined the effect of bending strain $\left(\varepsilon_{b}\right)$ on the transport $I_{c}$, magnetization loop width, on the MO images, and on the microstructure of 2223 tapes. Our study thus considerably amplifies earlier studies which covered only individual aspects of the study of bent tapes, ${ }^{9-13}$ to show that the method by which tapes are fabricated determines their response to strain. In particular, we found that the crack networks produced during the intermediate deformation steps are not completely healed by the subsequent heat treatments, and that the residual networks determine the subsequent response of tapes to bending.

Monofilament Ag-clad tape was made as described elsewhere. ${ }^{8}$ Samples were heat treated at $825^{\circ} \mathrm{C}$ in a flowing

\footnotetext{
a) On leave from the Institute of Electrical Engineering, Bratislava, Slovakia.

b) Also at the Institute of Solid State Physics, RAS, Chernogolovka, Moscow 142432, Russia.
}

$7.5 \% \mathrm{O}_{2} /$ balance $\mathrm{N}_{2}$ atmosphere, and were either uniaxially pressed or cold rolled to reduce their thickness by $10 \%-15 \%$ between heat treatments. Note that reductions of $10 \%-15 \%$ are clearly in the plastic regime, and must result in the formation of cracks in the BSCCO core. As mentioned above, ${ }^{8}$ the rolling and pressing processes produce characteristically different crack structures. The transport $J_{c}(77 \mathrm{~K}, 0 \mathrm{~T}, 1$ $\mu \mathrm{V} / \mathrm{cm})$ of the pressed sample was $15.2 \mathrm{kA} / \mathrm{cm}^{2}$, and the cross sectional area of the core was $\sim 8 \times 10^{-4} \mathrm{~cm}^{2}$. The rolled sample had $J_{c}=11 \mathrm{kA} / \mathrm{cm}^{2}$, and had the same cross sectional area as the pressed sample.

Straight samples were bent at room temperature to various diameters from 57 to $2.9 \mathrm{~mm}$, which correspond to maximum bending strains $\left(\varepsilon_{b}\right)$ of $0.09 \%-1.72 \%$ at the outside of the BSCCO core (where $\varepsilon_{b}=t / d, t$ is the core thickness and $d$ is the bend diameter). The samples were "double bend" at each diameter; that is, they were bent to a given diameter, bent back in the opposite sense to the same diameter, and then straightened for measurement. This double-bent procedure ensured that the damage caused by bending was distributed equally on both sides of the neutral axis. The $1 \mu \mathrm{V} / \mathrm{cm}$, $0 \mathrm{~T}$ critical currents and SQUID magnetization loops were both measured at $77 \mathrm{~K}$, with the field applied perpendicular to the broad face of the tape (i.e., nominally parallel to the $c$ axis of the 2223 grains) in the magnetization measurements. After electromagnetic measurement, the $\mathrm{Ag}$ was etched away from short sections of tape, and the crack structure visible on the broad surface of the bare core was examined by a scanning electron microscope (SEM). MO imaging of the magnetic flux penetration was performed by placing the MOsensitive film directly on top of the bare cores of the tape. ${ }^{8}$

Figure 1 plots the ratio $I_{c}\left(\varepsilon_{b}\right) / I_{c}(0)$, where $I_{c}\left(\varepsilon_{b}\right)$ the critical current of a sample bent to $\varepsilon_{b}$ and $I_{c}(0)$ is the critical current of the original, nonbent sample, as a function of bending strain, for both pressed and rolled samples, together with the ratio $\Delta M\left(\varepsilon_{b}\right) / \Delta M(0)$ for both pressed and rolled samples determined at external magnetic field $B_{c}=0$. For pressed samples, $I_{c}\left(\varepsilon_{b}\right) / I_{c}(0)$ declined by only $5 \%$ at $\varepsilon_{b}$ $\sim 0.2 \%$, but it then dropped sharply until $\varepsilon_{b} \sim 0.3 \%$, at which point $I_{c}$ had fallen to less than $30 \%$ of the original, 


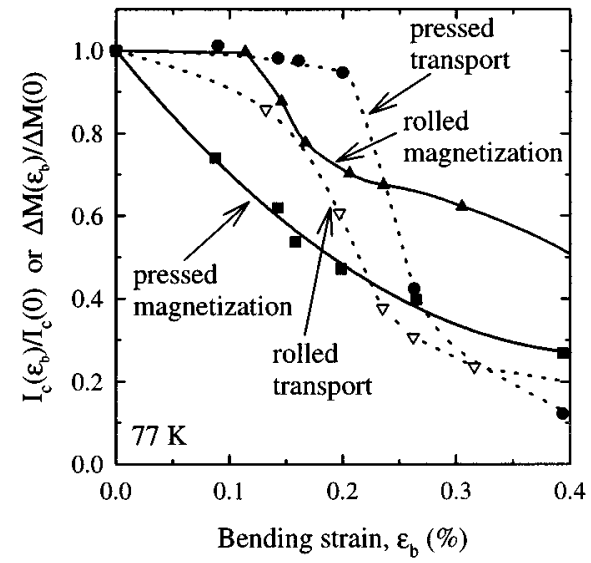

FIG. 1. Normalized transport critical current, $I_{c}\left(\varepsilon_{b}\right) / I_{c}(0)$, and magnetization, $\Delta M\left(\varepsilon_{b}\right) / \Delta M(0)$, as a function of bending strain for pressed and rolled samples. The transport and magnetization have different responses to strain.

undamaged sample. The current flowing in the Ag sheath was not taken into account in this analysis, as at $1 \mu \mathrm{V} / \mathrm{cm}$, it is much smaller than the current in the superconductor, and can be neglected within the measurement accuracy. The magnetization of pressed samples behaved much differently, decreasing continuously by $\sim 50 \%$ at $\varepsilon_{b} \sim 0.2 \%$. The transport current of rolled samples behaved in a manner between the pressed transport and magnetization results; it decreased by $40 \%$ for $\varepsilon_{b} \sim 0.2 \%$. The magnetization of rolled samples remained nearly unchanged up to $\varepsilon_{b} \sim 0.12 \%$, then decreased by $25 \%$ for $\varepsilon_{b}=0.2 \%$. At $\varepsilon_{b}=0.4 \%$, the magnetization was reduced to $50 \%$ of the original value, while $I_{c}$ was decreased to $20 \%$ of that of the original undamaged sample.

The MO images of a pressed, nonbent sample and a pressed sample bent to $\varepsilon_{b}=0.20 \%$ taken for a field of $40 \mathrm{mT}$ applied perpendicular to the broad tape face after cooling in zero field to $T=13 \mathrm{~K}$ are shown in Figs. 2(a) and 2(b), re- spectively. Brighter areas correspond to regions of higher magnetic flux density and thus lower shielding. The network of narrow bright channels within the core shows that flux enters the tape preferentially parallel to the long tape axis direction, as shown previously for pressed samples. ${ }^{8}$ We believe that such images reveal the residual microcrack network left after heat treatment. A bend strain of $0.2 \%$ appears to increase the prominence of this longitudinal flux penetration network (recall that bending also produces a transverse tensile strain), suggesting that damage propagates from this residual microcrack network [compare Figs. 2(a) and 2(b)]. However, no macrocracks caused by the bending are visible in Fig. 2(b), nor were they visible in SEM examination, in spite of the considerable decrease of the magnetization $\left[\Delta M\left(\varepsilon_{b}\right) / \Delta M(0)=0.5\right]$ that was observed at this strain (Fig. 1). This difference can be explained by considering that longitudinal cracks should influence the magnetization current more than the transport current, since transverse current flow is required to complete the magnetization current loops. Consistent with this interpretation, the transport critical current at $\varepsilon_{b}=0.20 \%$ was still the same as for the nonbent, $\varepsilon_{b}=0$ tape. The MO image of the pressed sample bent to $\varepsilon_{b}=0.26 \%$ is shown in Fig. 2(c). At this strain, long range, large macrocracks have propagated and are clearly visible (the bright barbed-wirelike lines which cross the tape are like those seen in x-ray imaging by Spal et al.), ${ }^{14}$ whereas no cracks were obvious in SEM pictures. In this case, the influence of the cracks was evident in the measurements of the transport critical current, as $I_{c}\left(\varepsilon_{b}\right) / I_{c}(0)$ was decreased to $\sim 0.4$ after bending to $\varepsilon_{b}=0.26 \%$. The MO results suggest why rolled samples behave differently: because the direction of residual cracks in rolled samples is transverse to the tape axis, as already clearly demonstrated by Parrell et al., ${ }^{8}$ any propagation of such cracks in deformed samples tends to sever the filament, ${ }^{15}$ and thus the transport behavior of rolled samples is more sensitive to even small bending strains, as

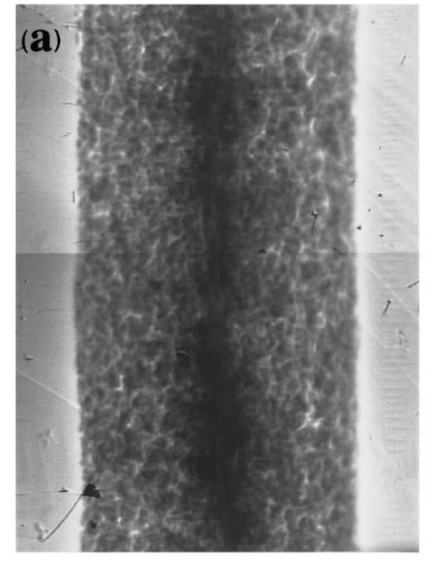

$\varepsilon_{\mathrm{b}}=0 \%$

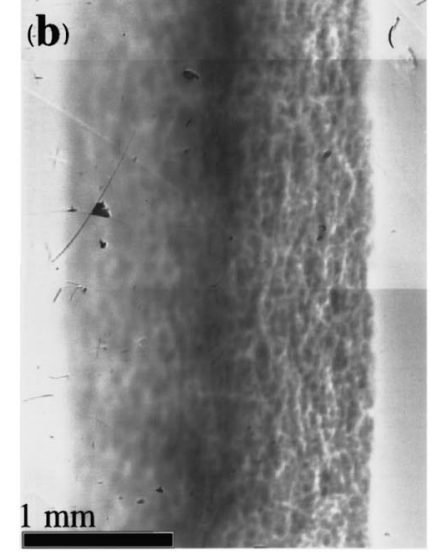

$\varepsilon_{\mathrm{b}}=0.20 \%$

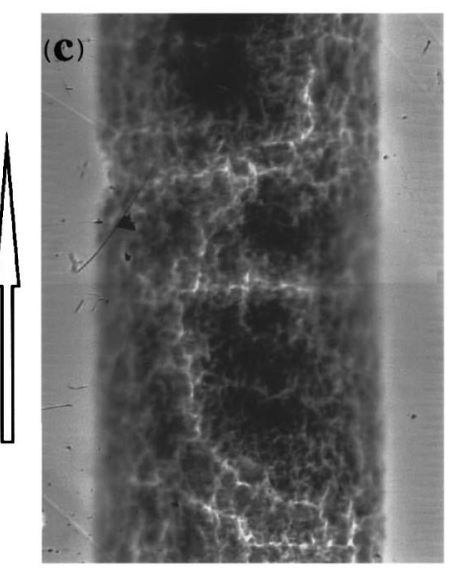

$\varepsilon_{\mathrm{b}}=0.26 \%$

FIG. 2. Magneto-optical images of the full width of some of the pressed samples taken at $B_{e}(\| c)=40 \mathrm{mT}$ after cooling in field to $13 \mathrm{~K}$. In these images, bright areas correspond to regions of higher magnetic flux density. The long tape axis is indicated by the vertical arrows. (a) is an image of a nonbent pressed sample, and (b) shows the $\varepsilon_{b}=0.20 \%$ pressed sample. There is a significant increase in the flux penetrating parallel to the tape axis on going from $\varepsilon_{b}=0$ to $\varepsilon_{b}=0.20 \%$. The left side of (b) has less contrast due to a slightly greater distance between the indicator film and the tape surface. (c) shows the $\varepsilon_{b}$ $=0.26 \%$ pressed sample. Obvious transverse and longitudinal cracks (the bright areas) are visible in this image. 


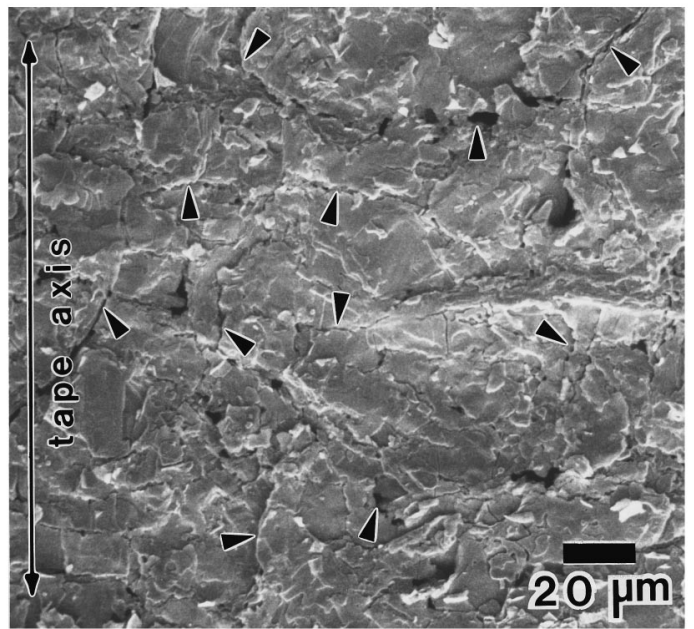

FIG. 3. SEM photomicrograph of the top surface of a pressed tape after bending to $\varepsilon_{b}=1.72 \%$. A few of the many visible cracks are indicated by arrowheads. The core has fractured into blocks of many grains.

shown in Fig. 1. The magnetization of the rolled sample is influenced by bending strain less than that of the pressed sample. Residual cracks oriented perpendicular to the tape axis exist in rolled BSCCC-2223 tapes, even in tapes that have not been bent. ${ }^{8}$ The formation of additional cracks by bending does not appreciably change the magnetization current loops within the pre-existing crack channel network, but they significantly reduce the transport current. Only for $\varepsilon_{b}$ $>0.12 \%$ do new cracks reduce the size of the magnetization current loops, and thus the total magnetization.

Although the orientation of the residual crack network plays a large role in determining the small bend strain behavior of pressed and rolled samples, it appears to influence the properties less at high values of bend strain, where many new cracks have been introduced, as evidenced by the very similar values of the normalized properties at $\varepsilon_{b}=0.4 \%$ in Fig. 1. Figure 3 is an SEM micrograph of the top surface of a pressed sample after it had been bent to $\varepsilon_{b}=1.72 \%$. This sample was the first in which cracks could consistently and generally be seen by SEM. Cracks are seen in transverse, longitudinal, and arbitrary directions, fragmenting the core into blocks of grains. Thus it is important to note that the total magnetization is not just the sum of the components due to "intergranular" currents which flow over the whole sample, and "intragranular" currents, which flow within individual grains, as is sometimes assumed. ${ }^{11}$ Several authors have shown that there is another intermediate length scale over which magnetization current loops flow. ${ }^{16,17}$ Based on the MO images of Fig. 2, and SEM images like that for the pressed $\varepsilon_{b}=1.72 \%$ sample of Fig. 3, one can expect magnetization contributions in bent samples from regions ranging from tens of microns, or many multiples of the grain size [Figs. 2(a), 2(b), and 3], to millimeters [Fig. 2(c)].

In summary, studies of the effect of bending strain on the transport critical current, magnetization, flux penetration, and microstructure of Ag-clad monofilament BSCCO-2223 tapes have revealed that the transport critical current re- mained essentially unchanged until a bending strain of about $0.2 \%$ occurred for pressed samples, then decreased sharply, whereas the magnetization signal continuously and smoothly decreased with very small bending strains. For bending strains greater than $\sim 0.3 \%$, the width of the magnetization curves becomes saturated at a value of $\sim 25 \%$ of the original value, while the transport critical currents become nearly zero. In contrast to pressed samples, the critical currents of samples rolled between heat treatments began to decrease with even very small strains, like the pressed sample magnetization, and then decreased more sharply at a strain of $\sim 0.15 \%$. The magnetization of rolled samples is less sensitive to bending strain, as the residual cracks are oriented perpendicular to the tape axis. We attribute these behaviors to different patterns of the residual, preferentially oriented crack networks in rolled and pressed tapes, and the different paths of transport and magnetization currents. These experiments clearly show that residual thermomechanical processing deformation damage makes a significant impact on the superconducting properties measured as a function of bend strain, and are further evidence that cracks produced during thermomechanical processing are difficult to entirely close. These results stress the importance of the intergranular connectivity as an important critical current density limitation in BSCCO-2223 tapes. They also underscore the great value of magneto-optical imaging as a means of viewing the location of current limiting defects.

Discussions with various members of the Wire Development Group are gratefully acknowledged. This work was supported by ARPA (N00014-90 J-4115) and EPRI (RP8009-05).

${ }^{1}$ D. C. Larbalestier, S. E. Babcock, X. Y. Cai, S. E. Dorris, H. S. Edelman, A. Gurevich, J. A. Parrell, A. Pashitski, M. Polak, A. Polyanskii, I.-F. Tsu, and J.-L. Wang, Appl. Supercond. 1, 29 (1995).

${ }^{2}$ D. C. Larbalestier, Proceedings of the 10th Anniversary HTS Workshop on Physics, Materials, and Applications, Houston, March 12-16 (unpublished), 1996.

${ }^{3}$ B. A. Glowacki and J. Jackiewizc, J. Appl. Phys. 75, 2992 (1994).

${ }^{4}$ J. A. Parrell, S. E. Dorris, and D. C. Larbalestier, Physica C 231, 137 (1994).

${ }^{5}$ D. C. Larbalestier, Y. Feng, X. Y. Cai, H. Edelman, E. E. Hellstom, Y. E. High, J. A. Parrell, Y. S. Sung, and A. Umezawa, in Proceedings of the 7th International Workshop of Critical Currents in Superconductors, edited by H. W. Weber (World Scientific, Singapore, 1994), p. 82.

${ }^{6}$ D. A. Korzekwa, J. F. Bingert, E. J. Podtburg, and P. Miles, Appl. Supercond. 2, 26 (1994).

${ }^{7}$ M. Lahtinen, J. Paasi, J. Sarkaniemi, Z. Han, and T. Freltoft, Physica C 244, 115 (1995).

${ }^{8}$ J. A. Parrell, A. A. Polyanskii, A. E. Pashitski, and D. C. Larbalestier, Semicond. Sci. Technol. 9, 393 (1996).

${ }^{9}$ W. D. Lee, L. Horng, T. J. Yang, and B. S. Chiou, Physica C 247, 215 (1995).

${ }^{10}$ P. Kovac, I. Husek, and L. Cesnak, Semicond. Sci. Technol. 7, 583 (1994).

${ }^{11}$ K. H. Müller, C. Andrikidis, H. K. Liu, and S. X. Dou, Phys. Rev. B 50, 10218 (1994).

${ }^{12}$ M. Lahtinen, J. Paasi, J. Sarkaniemi, Z. Han, and T. Freltoft (unpublished).

${ }^{13}$ M. Suenaga, Y. Fukumoto, P. Haldar, T. R. Thurston, and U. Wildgrüber, Appl. Phys. Lett. 67, 3025 (1995).

${ }^{14}$ R. D. Spal, G. N. Riley, Jr., and C. Christopherson (unpublished).

${ }^{15}$ A. Polyanskii, A. Pashitski, A. Gurevich, J. A. Parrell, M. Polak, D. C. Larbalestier, S. R. Foltyn, and P. N. Arendt, Proceedings of ISS'96, Sapporo, Japan, 21-23 October 1996.

${ }^{16}$ J. Paasi, A. Tuohimaa, and J.-T. Eriksson, Physica C 259, 10 (1996).

${ }^{17}$ A. E. Pashitski, A. Polyanskii, A. Gurevich, J. A. Parrell, and D. C. Larbalestier, Appl. Phys. Lett. 67, 2720 (1995). 